\title{
Shape-selective separation of polycyclic aromatic hydrocarbons by reversed-phase liquid chromatography on tetraphenylporphyrin-based stationary phases
}

\author{
Chris E. Kibbey and Mark E. Meyerhoff* \\ Department of Chemistry, University of Michigan, Ann Arbor, MI 48109 (USA)
}

(First received December 18th, 1992; revised manuscript received March 9th, 1993)

\begin{abstract}
The reversed-phase chromatographic behavior of planar and non-planar polycyclic aromatic hydrocarbons (PAHs) is investigated on tetraphenylporphyrin and two metallotetraphenylporphyrin [Sn(IV), In(III)] bonded stationary phases using methanol-water and acetonitrile-water as mixed solvent mobile phases. Large differences in the capacity factors of aromatic solute pairs having the same number of carbon atoms, but differing in three-dimensional shape (e.g., triphenylene/o-terphenyl and perylene $/ \alpha, \alpha^{\prime}$-binaphthyl), suggest that the three tetraphenylporphyrin-based supports possess shape selectivity toward small planar aromatic solutes. Capacity factors for planar PAH solutes on these supports are significantly greater than for non-planar polyaryls having the same number of carbon atoms.
\end{abstract}

\section{INTRODUCTION}

Polycyclic aromatic hydrocarbons (PAHs) are ubiquitous in the environment, as they are formed readily by the combustion of virtually any organic material. Within the general class of PAHs, the most virulent carcinogens are those planar PAHs which possess potent bay region activity [1]. While the link between the angular arrangement of a PAH's fused rings and its carcinogenic activity has been well established [2], the development of chromatographic stationary phases having adequate shape selectivity to discriminate between planar and non-planar PAHs remains a challenge.

Reversed-phase liquid chromatography is the method of choice for the separation and quantitation of PAHs. Reversed-phase separations of PAHs typically are performed on chemically bonded octadecylsilyl (ODS) phases [3]. The

\footnotetext{
* Corresponding author.
}

morphology of bonded ODS groups on the surface of these supports varies depending on the functionality of the alkyl silane used. Commercial ODS phases prepared from monofunctional silanes yield a brush-like monomeric coverage of octadecyl chains on the silica surface, while polyfunctional silanes are used to prepare ODS phases with a comparatively more rigid network of octadecyl chains.

The morphology of the bonded ODS groups plays a significant role in PAH planarity recognition on these supports. The greater shape selectivity of polymeric ODS phases makes these supports better suited than monomeric ODSsilicas for PAH separations [4]. Shape selectivity on polymeric ODS supports is attributed to the ability of planar solutes to slide more readily into "slots" formed by the rigid network of ordered alkyl chains than do non-planar solutes $[5,6]$. The alkyl chains of monomeric ODS phases are able to adopt a more random orientation on the silica surface; hence these supports are less able to discriminate between planar and non-planar 
solutes on the basis of shape. Additional evidence that solute shape selectivity is influenced by the morphology of alkyl chains on the stationary phase comes from the observation that the chromatographic properties of solutes on monomeric straight-chain alkyl phases become more like those of polymeric stationary phases as the chain lengths of the bonded alkanes are increased $[7,8]$, the bonding density of alkyl chains on the silica surface is increased [9], or the stationary phase temperature is decreased [10]. In the latter case, alkyl chain mobility decreases with decreasing temperature, resulting in a bonded phase morphology that resembles that of a crystalline solid [10]. Long alkyl chains adopt a more ordered arrangement on the silica surface due to configurational constraints than short alkyl chains [7]; yielding a surface morphology for the former characterized by a comparatively rigid network of deep slots. In addition, the alkyl chain packing structure on the silica surface becomes more ordered as the surface bonding density is increased [9].

Several sets of PAH solutes have been used to characterize the shape selectivity of reversedphase supports [4,11]. Among these are the set consisting of phenanthro[3,4-c]phenanthrene (PhPh), a helically shaped PAH; benzo[ $a]$ pyrene (BaP), a planar solute; and dibenzo[ $g, p]$ chrysene (TBN), which is saddle shaped. The elution order of these three solutes on a monomeric ODS support is: $\mathrm{TBN}>\mathrm{PhPh}>\mathrm{BaP}$; while on a polymeric ODS support the order of elution is: $\mathrm{BaP}>\mathrm{TBN}>\mathrm{PhPh}$ [4]. Other investigators have used aromatic solute pairs, which have the same number of carbon atoms, but differ in three-dimensional shape (i.e., triphenylene/o-terphenyl), to assess the shape selectivity of reversed-phase supports [11].

Separations of small PAHs (2-5 rings) on a number of aromatic hydrocarbon bonded stationary phases, including phenyl, $p$-bis(dimethylphenyl), 1,3,5-tris(trimethylphenyl), naphthylethyl, and pyrenylethyl, have also been reported [11-13]. The dominant factors affecting retention on these supports are the size and shape of the PAH solute. Retention on these supports is believed to result from a combination of $\pi-\pi$ and shape interactions between the solute PAHs and the surface-bonded aromatic groups. PAH retention and stationary phase shape selectivity increase with the size of the chemically bonded aromatic system [11]. In contrast to the usual shape selectivity observed with polymeric ODS phases, both the $p$-bis(dimethylphenyl) and the 1,3,5-tris(trimethylphenyl) bonded phases displayed greater selectivity for non-planar versus planar solutes due to an apparent steric constraint between planar solutes and pendant methyl groups on the bonded phenyl moiety [12].

Recently, we reported the synthesis and characterization of silica gel stationary phases possessing immobilized tetraphenylporphyrin and metallotetraphenylporphyrins [Sn(IV) and In(III)] [14]. The two chemically bonded metallotetraphenylporphyrin-silicas proved useful for the anion-exchange separation of aromatic sulfonates and carboxylates. The presence of a macrocyclic aromatic system in tetraphenylporphyrin [15] prompted preliminary investigations into the possible application of these stationary phases to the separation of PAHs [14]. We report here, in more detail, our observations regarding the reversed-phase separation of several small PAHs (2-4 rings) on 5-paracarboxyphenyl 10,15,20phenylporphyrin- ( $\mathbf{H}_{2}$ TPP), and In(III) and Sn(IV) metallated 5-para-carboxyphenyl 10,15,20-tri-phenylporphyrin- [In(TPP) and Sn(TPP), respectively] bonded stationary phases. For small PAHs, these three tetraphenylporphyrin-silicas yield a linear relationship between the logarithm of the capacity factor $\left(k^{\prime}\right)$ and the correlation factor $(F)$. Differences in aromatic solute retention between the tetraphenylporphyrin and polymeric ODS supports as a function of temperature are also discussed.

\section{EXPERIMENTAL}

\section{Equipment}

The HPLC system used in this work consisted of a Spectra-Physics (San Jose, CA, USA) SP 8700 solvent-delivery system, a Spectra-Physics SP 4290 computing integrator, a Kratos (Ramsey, NJ, USA) Spectroflow 773 variable-wavelength UV-Vis detector, an ISCO (Lincoln, NE, USA) ISIS autosampler and a Rheodyne 
(Cotati, CA, USA) model 7010 sample valve with a $20-\mu$ l loop. The columns were thermostated using a Fisher Scientific (Pittsburgh, PA, USA) water jacket connected to a Fisher Scientific Model 80 Isotemp constant-temperature circulator.

\section{Materials}

The preparation of the $\mathrm{H}_{2}$ TPP, In(TPP) and $\mathrm{Sn}$ (TPP) stationary phases used in this work is described elsewhere [14]. The three tetraphenylporphyrin silicas were slurry packed in methanol-water $(50: 50, \mathrm{v} / \mathrm{v})$ by the down-fill method [16] into separate $10 \mathrm{~cm} \times 4.6 \mathrm{~mm}$ I.D. stainlesssteel columns (Alltech, Deerfield, IL, USA). The aromatic solutes were of reagent grade or better and purchased from Aldrich (Milwaukee, WI, USA); with the exception of $\alpha, \alpha^{\prime}-$ binaphthyl, which was obtained from ICN (Irvine, CA, USA). The mobile phases used in this work were made up by volume from LC-grade solvents (Mallinckrodt, Paris, KY, USA) and 16 $\mathbf{M} \Omega$ deionized water.

\section{RESULTS AND DISCUSSION}

The porphyrin coverage on the $\mathrm{H}_{2} \mathrm{TPP}$, In(TPP) and Sn(TPP) supports was determined from the increase in nitrogen content of the aminopropyl silica starting material following tetraphenylporphyrin immobilization, acetylation with acetic anhydride, and metallation of the immobilized tetraphenylporphyrin with either tin(IV) or indium(III) [14]. The nitrogen content and porphyrin coverage of the three supports are shown in Table I. Both the $\mathrm{H}_{2}$ TPP and the In(TPP) phases have similar porphyrin coverages ( 0.35 and $0.32 \mu \mathrm{mol} / \mathrm{m}^{2}$, respectively), while the

\section{TABLE I}

SURFACE PORPHYRIN COVERAGE ON THE THREE PORPHYRIN-SILICAS

\begin{tabular}{lll}
\hline Porphyrin phase & Nitrogen (\%) & $\begin{array}{l}\text { Porphyrin coverage } \\
\left(\mu \mathrm{mol} / \mathrm{m}^{2}\right)\end{array}$ \\
\hline H$_{2}$ TPP & 0.98 & 0.35 \\
InTPP & 0.93 & 0.32 \\
SnTPP & 1.48 & 0.18 \\
\hline
\end{tabular}

porphyrin coverage on the $\mathrm{Sn}(\mathrm{TPP})$-silica (0.18 $\mu \mathrm{mol} / \mathrm{m}^{2}$ ) is lower. The $\mathrm{Sn}(\mathrm{TPP})$ phase was prepared from an aminopropyl silica with a smaller pore size and larger surface area than that used to prepare the $\mathrm{H}_{2}$ TPP and In(TPP) supports. Consequently, in the preparation of the $\mathrm{Sn}(\mathrm{TPP})$-silica, the porphyrin may have been excluded from access to the amine sites on the interior surface of the aminopropyl silica.

The efficiencies of the three columns packed with the tetraphenylporphyrin-silicas were determined from the elution of a toluene band with a mobile phase consisting of methanol-water $(50: 50, v / v)$. Column efficiency was calculated from the retention time of the toluene peak $\left(t_{\mathrm{R}}\right)$ and its peak width at baseline $\left(t_{\mathrm{w}}\right)$ as follows:

$N=16\left(\frac{t_{\mathrm{R}}}{t_{\mathrm{w}}}\right)^{2}$

The $\mathrm{H}_{2}$ TPP, In(TPP) and Sn(TPP) columns had average $(n=4)$ efficiencies of 2100,2200 and 2900 theoretical plates, respectively. While these column efficiencies are poorer than those of commercially packed columns, the efficiencies obtained here were sufficient for evaluating the separation of PAHs. Further, the slight asymmetry of the solute bands eluting from these columns is attributed to poor stationary phase packing (see Fig. 4).

The capacity factors of nine PAH solutes on the three tetraphenylporphyrin-based supports using methanol-water and acetonitrile-water mobile phases of different composition are presented in Table II. While the In(TPP) stationary phase exhibits slightly greater retention of triphenylene, chrysene and perylene than the $H_{2}$ TPP and the $\mathrm{Sn}$ (TPP) silicas, there is no difference in the elution order of the aromatic solutes on the three tetraphenylporphyrin-silica stationary phases. It is important to note that the capacity factors of the PAHs were negligible on a column packed with acetylated aminopropyl silica alone (data not shown). Interactions between these aromatic solutes and acetylated aminopropyl groups or residual silanol sites on the support surface do not appear to play a role in solute retention on the three tetraphenylporphyrin-silicas. 
TABLE II

PAH CAPACITY FACTORS ON THE THREE PORPHYRIN-SILICAS UNDER THE MOBILE PHASE CONDITIONS INDICATED

\begin{tabular}{|c|c|c|c|c|c|c|c|c|c|c|c|c|}
\hline \multirow[t]{3}{*}{ PAH solute } & \multicolumn{12}{|c|}{ Capacity factor $\left(k^{\prime}\right)$} \\
\hline & \multicolumn{3}{|c|}{ Methanol-water (80:20) } & \multicolumn{3}{|c|}{ Methanol-water (70:30) } & \multicolumn{3}{|c|}{ Acetonitrile-water $(60: 40)$} & \multicolumn{3}{|c|}{ Acetonitrile-water $(50: 50)$} \\
\hline & $\mathrm{H}_{2} \mathrm{TPP}$ & InTPP & SnTPP & $\mathrm{H}_{2} \mathrm{TPP}$ & InTPP & SnTPP & $\mathrm{H}_{2} \mathrm{TPP}$ & InTPP & SnTPP & $\mathrm{H}_{2} \mathrm{TPP}$ & InTPP & SnTPP \\
\hline Naphthalene & 0.75 & 0.72 & 0.74 & 1.26 & 1.13 & 1.40 & 0.87 & 0.73 & 0.73 & 1.66 & 1.53 & 1.62 \\
\hline Phenanthrene & 1.80 & 1.76 & 1.76 & 3.71 & 3.58 & 3.99 & 1.59 & 1.54 & 1.38 & 3.56 & 3.73 & 3.40 \\
\hline Anthracene & 1.94 & 1.90 & 1.85 & 4.12 & 3.94 & 4.24 & 1.69 & 1.64 & 1.49 & 3.85 & 4.04 & 3.56 \\
\hline Pyrene & 3.17 & 3.27 & 3.14 & 7.03 & 7.32 & 7.53 & 2.56 & 2.98 & 2.22 & 6.08 & 7.49 & 5.41 \\
\hline Chrysene & 5.16 & 5.79 & 4.96 & 12.74 & 15.34 & 13.24 & 3.49 & 4.20 & 3.08 & 9.09 & 11.68 & 8.02 \\
\hline$o$-Terphenyl & 1.54 & 1.31 & 1.58 & 3.46 & 2.83 & 3.97 & 1.59 & 1.26 & 1.37 & 4.04 & 3.49 & 3.73 \\
\hline Triphenylene & 4.84 & 5.62 & 4.76 & 12.04 & 14.49 & 12.48 & 3.24 & 3.85 & 2.94 & 8.24 & 10.52 & 7.64 \\
\hline$\alpha, \alpha^{\prime}$-Binaphthyl & 3.03 & 2.40 & 3.09 & - & - & - & 2.42 & 2.44 & 2.28 & - & - & - \\
\hline Perylene & 11.87 & 15.46 & 10.56 & - & - & - & 6.40 & 6.42 & 5.73 & - & - & - \\
\hline
\end{tabular}

There are, however, significant differences in PAH retention observed on all three supports as the mobile phase is changed from one that is methanol based to one that is based on acetonitrile. A lower mobile phase concentration of acetonitrile is needed to achieve a given PAH capacity factor on the tetraphenylporphyrinbased supports compared to a methanol-based mobile phase. Acetonitrile generally is regarded as a stronger eluent than methanol, as at a given composition, solute retention on reversed-phase supports is greater with methanol-water than with acetonitrile-water eluents. However, when dealing with stationary phases and solutes possessing unsaturated sites, the influence of $\pi-\pi$ interactions (between the cyano group of acetonitrile and the double bonds of the solute and stationary phase) on solute retention must be considered in addition to solvophobic effects [17].

The appreciable difference in capacity factors between $o$-terphenyl/triphenylene and $\alpha, \alpha^{\prime}$-binaphthyl/perylene on the three porphyrin-silicas (Table II) indicates that these stationary phases possess selectivity for planar versus non-planar solutes. These aromatic solute pairs were chosen for study based on their commercial availability and prior use as probes for assessing shape selectivity [11]. The ratio of the capacity factors of triphenylene and $o$-terphenyl $\left(\alpha_{\text {trip/o-ter }}\right)$ on the $\mathrm{H}_{2}$ TPP and $\mathrm{Sn}$ (TPP) columns is approximately 3 when methanol-based mobile phases are used, while on the In(TPP) column, this ratio is approximately 4 . The selectivity factors for the perylene $/ \alpha, \alpha^{\prime}$-binaphthyl pair $\left(\alpha_{\text {pery/a, }}\right)$ on the $\mathrm{H}_{2}$ TPP-, In(TPP)- and Sn(TPP)-based supports with a methanol-water (80:20) mobile phase $(3.9,6.4$ and 3.4 , respectively) are even greater than those for the triphenylene/o-terphenyl pair on the three tetraphenylporphyrinsilicas. On a typical monomeric ODS stationary phase the selectivity factor ( $\left.\alpha_{\text {trip/o-ter }}\right)$ ranges from 1.0 to 1.7 [13], while the value of $\alpha_{\text {trip/o-ter }}$ on a polymeric ODS phase lies between 2.0 and 2.7 [6]. Jinno et al. [13] suggest that a selectivity factor $\left(\alpha_{\text {trip/o-rer }}\right)$ of 2.0 or greater is an indication of significant solute planarity recognition. Based on this latter criterion, the three tetraphenylporphyrin-silicas possess a high degree of shape selectivity for small aromatic solutes.

While both polymeric ODS and tetraphenylporphyrin-silica phases display selectivity for planar solutes, we believe that the retention mechanisms that lead to shape selectivity on these two types of support may be different. According to the "slot model", interactions with polymeric ODS supports that result in increased retention of planar solutes are primarily physical in nature [5]. Shape selectivity on the tetraphenylporphyrin-silicas, however, may reflect differences in the magnitudes of $\pi-\pi$ overlap of aromatic solutes with the tetraphenylporphyrin macrocycle. For example, the aromatic macrocycle of tetraphenylporphyrin is large enough to 
(A)

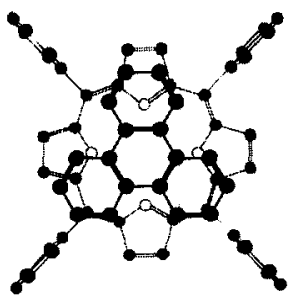

(B)
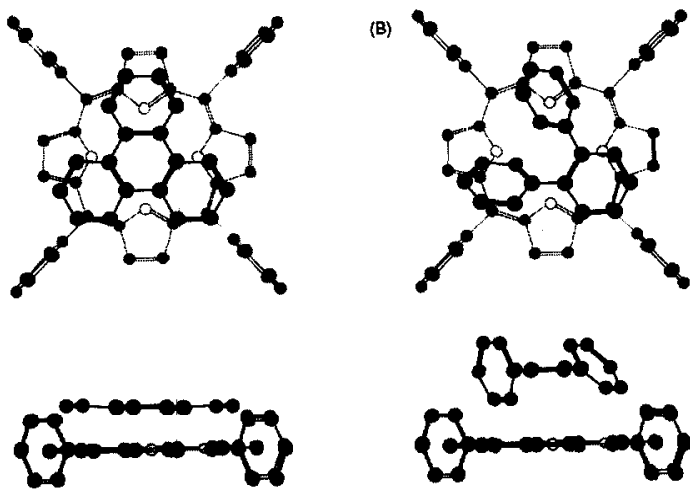

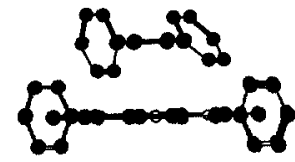

Fig. 1. Idealized interactions of (A) triphenylene and (B) $o$-terphenyl with the tetraphenylporphyrin macrocycle.

accommodate both triphenylene and $o$-terphenyl (Fig. 1). However, the non-planar geometry of the latter prevents it from attaining as strong an interaction with the tetraphenylporphyrin macrocycle as triphenylene. A similar argument can be made to account for the greater retention of perylene compared to $\alpha, \alpha^{\prime}$-binaphthyl on the three tetraphenylporphyrin-silicas (Table II). Of course, a more thorough examination of the retention of PAH solutes differing in three-dimensional shape (e.g., $\mathrm{PhPh}, \mathrm{TBN}$ and $\mathrm{BaP}$ ) will be required before more definitive conclusions regarding shape sclectivity on these supports can be drawn. It should also be noted that Fig. 1 is meant to convey the idealized differences in $\pi-\pi$ interaction of the two solutes with the tetraphenylporphyrin macrocycle, and not necessarily an accurate model of such interactions.

Shape selectivity on pyrenylethyl-silica has been attributed to the ability of planar solutes to partition into slots formed from the closely spaced arrangement of aggregated pyrenylethyl groups on the silica surface [11]. The likelihood of a similar closely spaced arrangement of tetraphenylporphyrin groups on the three tetraphenylporphyrin-silicas used here seems unlikely for two reasons. First, the bonding density of aminopropyl groups on the starting silica phase was approximately one half of its theoretical maximum value [14]. Consequently, the density of tetraphenylporphyrins immobilized on the surface of these supports is low. Furthermore, tetraphenylporphyrins generally do not aggregate due

to the near perpendicular orientation of their meso-phenyl rings to the porphyrin macrocycle [18].

Increasing the temperature at which a particular separation is carried out in LC generally results in a decrease in the separation factor $(\alpha)$ between pairs of solutes, but does not change the solute elution order. Snyder refers to such behavior as a "regular" temperature effect [19]. However, "irregular" temperature effects have been observed for solutes differing in three-dimensional shape on ODS supports. That is, changes in the separation temperature of "irregular" systems not only result in changes in $\alpha$, but in changes in the elution order of the solutes as well. For example, the elution order of the solute pairs $o$-terphenyl/anthracene and $\alpha, \alpha^{\prime}$-binaphthyl/triphenylene is reversed on an ODS support as the column temperature is increased [20]. In addition, the elution order of the three $\mathrm{PAH}$ probes $(\mathrm{PhPh}, \mathrm{TBN}$ and $\mathrm{BaP})$ is influenced by column temperature on polymeric ODS supports [10]. Snyder [19] argues that at higher temperatures solute retention on ODS supports is favored by solutes that are more compact and near-spherical in three-dimensional shape.

Dissimilarities between ODS and the new tetraphenylporphyrin-silica supports are apparent from differences in the effects of column temperature on the elution order of small aromatic solutes. Four aromatic solutes (o-terphenyl, triphenylene, anthracene and $\alpha, \alpha^{\prime}$-binaphthyl) were eluted from the $\operatorname{In}(\mathrm{TPP})$-silica support with methanol-water (70:30) at column temperatures ranging from 25 to $65^{\circ} \mathrm{C}$. A plot of $\ln k^{\prime}$ versus reciprocal temperature $(1 / T)$ for these four aromatic solutes is shown in Fig. 2. The linear variation in $\ln k^{\prime}$ as a function of $1 / T$ for these four solutes indicates that their individual retentions are governed by a single sorption mechanism. Furthermore, it is apparent that the selectivity factor $(\alpha)$ between pairs of solutes decreases as the temperature is increased, but their elution order on In(TPP)-silica does not change (Fig. 2). Thus, "regular" temperature effects are observed with these aromatic solutes on In(TPP)-silica whereas the elution of these solutes on ODS-silica is "irregular" with respect to temperature [20]. 


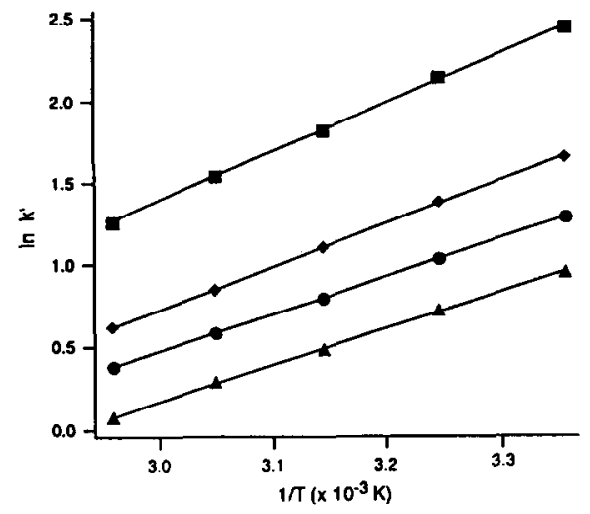

Fig. 2. Plot of $\ln k^{\prime}$ versus $1 / T$ for (D) triphenylene, (A) $o$-terphenyl, $(\diamond) \alpha, \alpha^{\prime}$-binaphthyl and (O) anthracene on In(TPP)-silica. Mobile phase: methanol-water (70:30).

The linear correlation between $\log k^{\prime}$ and the number of double bonds in an unsubstituted PAH [correlation factor $(F)$ ] is well established for ODS supports [21]. A plot of $\log k^{\prime}$ versus correlation factor for the elution of napthalene $(F=5)$, anthracene $(F=7)$, pyrene $(F=8)$ and chrysene $(F=9)$ from the three tetraphenylporphyrin-silica packed columns with a methanolwater (70:30) mobile phase is shown in Fig. 3. A linear relationship between $\log k^{\prime}$ and $F$ is also apparent for these PAH solutes on the three tetraphenylporphyrin-silicas.

Separation of five PAHs on the three porphyrin-silicas are shown in the three chromato-

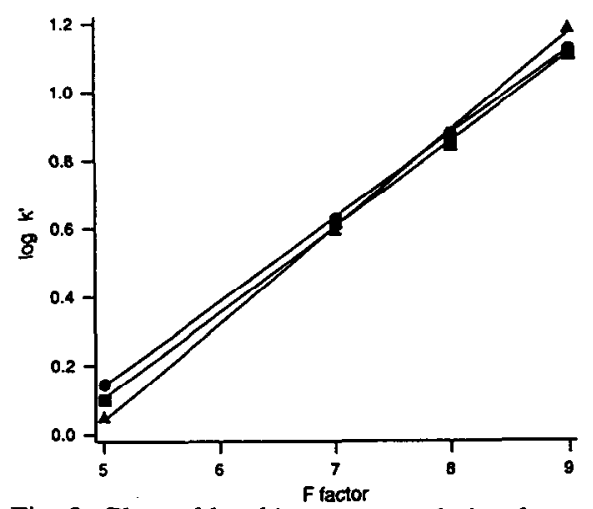

Fig. 3. Plots of $\log k^{\prime}$ versus correlation factor $(F)$ for PAHs on the (G) $\mathrm{H}_{2}$ TPP, (A) InTPP and (O) SnTPP bonded supports. Linear regresion analysis: $\mathrm{H}_{2}$ TPP (slope: 0.25 , intercept: -1.14 ); InTPP (slope: 0.28, intercept: -1.36 ); and SnTPP (slope: 0.24 , intercept: -1.08 ). grams of Fig. 4. The five solutes are reasonably well separated on all three stationary phases using a mobile phase of methanol-water (70:30). The elution order is similar to the elution order of these solutes on conventional ODS bonded phases. The separation of phenanthrene and anthracene on the three tetraphenylporphyrinsilicas could be improved through more efficient stationary phase packing and through the use of columns longer than the $10-\mathrm{cm}$ analytical columns used in this work. The macrocyclic ring of the tetraphenylporphyrin system is wide enough to accommodate most PAHs containing up to 4 or 5 rings (Fig. 1). Allowing for rotation of the immobilized tetraphenylporphyrin's four meso-phenyl rings from their near perpendicular orientation to the porphyrin macrocycle, PAHs

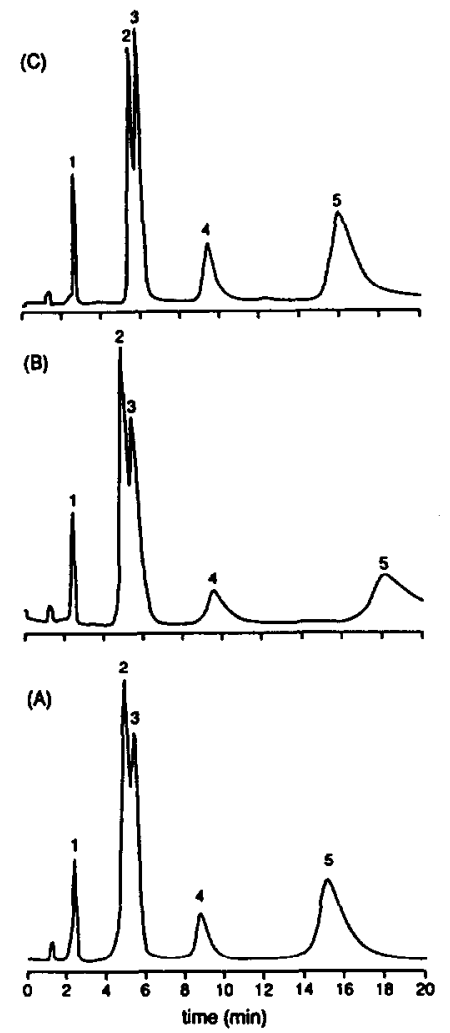

Fig. 4. Separation of five PAHs on the (A) $\mathrm{H}_{2}$ TPP, (B) InTPP and (C) SnTPP columns. Peaks: $1=$ naphthalene; $2=$ phenanthrene; $3=$ anthracene; $4=$ pyrene; $5=$ chrysene. Mobile phase: methanol-water (70:30). Flow-rate: $1.0 \mathrm{ml} /$ min. Column temperature: $25^{\circ} \mathrm{C}$. Detection: $254 \mathrm{~nm}(0.100$ AUFS). 
made up of more than 5 aromatic rings conceivably could also be separated on these tetraphenylporphyrin-silicas via preferential $\pi-\pi$ interaction with the porphyrin macrocycle.

\section{CONCLUSIONS}

Tetraphenylporphyrins chemically bonded onto silica yield stationary phases with novel shape selectivity toward PAHs. The greater retention of planar (versus non-planar) aromatic solutes on these supports may reflect greater $\pi-\pi$ overlap between the former and the porphyrin macrocycle. We are currently evaluating the chromatographic behavior of larger PAH solutes on these supports. In addition, we plan on studying the elution of other PAHs having dissimilar three-dimensional shape in order to gain a better understanding of the apparent shape selectivity of these supports. Performing these separations with normal-phase eluents (e.g., hexane-dichloromethane) may help to distinguish between solute retention based on solvophobic versus $\pi-\pi$ interactions. Further, there are other classes of macrocyclic aromatic compounds, such as phthalocyanine, sapphyrin and rubyrin, that also may be used to prepare LC stationary phases with interesting solute shape selectivities.

\section{ACKNOWLEDGEMENTS}

The authors gratefully acknowledge partial financial suport for this work from the National Institutes of Health (GM 28882). C.E.K. also acknowledges financial support from Amoco and Sokol funded Department of Chemistry fellowships. In addition, we thank the Anspec Co. (Ann Arbor, MI, USA) for use of their column packing equipment.

\section{REFERENCES}

1 G.H. Loew, J. Phillips, J. Wong, L. Hjelmeland and G. Pack, Cancer Biochem. Biophys., 2 (1978) 113-122.

2 R.G. Harvey, Polycyclic Aromatic Hydrocarbons: Chemistry and Carcinogenicity, Cambridge University Press, New York, 1991, Ch. 3.

3 K. Ogan, E. Katz and W. Slavin, Anal. Chem., 51 (1979) 1315-1320.

4 L.C. Sander and S.A. Wise, Anal. Chem., 56 (1984) 504-510.

5 S.A. Wise and L.C. Sander, J. High Resolut. Chromatogr. Chromatogr. Commun., 8 (1985) 248-255.

6 K. Jinno, T. Ibuki, N. Tanaka, M. Okamoto, J.C. Fetzer, W.R. Biggs, P.R. Griffiths and J.M. Olinger, J. Chromatogr., 461 (1989) 209-227.

7 L.C. Sander and S.A. Wise, Anal. Chem., 59 (1987) 2309-2313.

8 N. Tanaka, K. Sakagami and M. Araki, J. Chromatogr., 199 (1980) 327-337.

9 K.B. Sentell and J.G. Dorsey, J. Chromatogr., 461 (1989) 193-207.

10 L.C. Sander and S.A. Wise, Anal. Chem., 61 (1989) 1749-1754.

11 N. Tanaka, Y. Tokuda, K. Iwaguchi and M. Araki, J. Chromatogr., 239 (1982) 761-772.

12 K. Jinno, T. Nagoshi, N. Tanaka, M. Okamoto, J.C. Fetzer and W.R. Biggs, J. Chromatogr., 386 (1987) 123135.

13 K. Jinno, K. Yamamoto, H. Nagashima, T. Ueda and K. Itoh, J. Chromatogr., 517 (1990) 193-207.

14 C.E. Kibbey and M.E. Meyerhoff, Anal. Chem., in press (1993).

15 S.J. Silvers and A. Tulinsky, J. Am. Chem. Sac., 89 (1967) 3331-3337.

16 C.F. Poole and S.A. Schuette, Contemporary Practice of Chromatography, Elsevicr, New York, 1984, Ch. 4.

17 G. Thévenon-Emeric, A. Tchapla and M. Martin, J. Chromatogr., 550 (1991) 267-283.

18 W.I. White, in D. Dolphin (Editor), The Porphyrins, Academic Press, New York, 1978, Vol. V, Part C, Ch. 7.

19 L.R. Snyder, J. Chromatogr., 179 (1979) 167-172.

20 J. Chmielowiec and H. Sawatzky, J. Chromatogr. Sci., 17 (1979) 245-252.

21 J.F. Schabron, R.J. Hurtubise and H.F. Silver, Anal. Chem., 49 (1977) 2253-2260. 